\title{
Assessment of the ellipsoidal-statistical Bhatnagar-Gross-Krook model for force-driven Poiseuille flows
}

\author{
Jianping Meng, Lei Wu, Jason M. Reese, Yonghao Zhang* \\ James Weir Fluids Lab, Department of Mechanical \& Aerospace Engineering, University of Strathclyde, Glasgow G1 1XJ, UK
}

\section{A R T I C L E I N F O}

\section{Article history:}

Received 6 July 2012

Received in revised form 23 May 2013

Accepted 24 May 2013

Available online 13 June 2013

\section{Keywords:}

Kinetic theory

Gas dynamics

BGK model

ES-BGK model

$\mathrm{S}$ model

\begin{abstract}
A B S T R A C T
We investigate the accuracy of the ellipsoidal-statistical Bhatnagar-Gross-Krook (ES-BGK) kinetic model for planar force-driven Poiseuille flows. Our numerical simulations are conducted using the deterministic discrete velocity method, for Knudsen numbers (Kn) ranging from 0.05 to 10 . While we provide numerically accurate data, our aim is to assess the accuracy of the ES-BGK model for these flows. By comparing with data from the direct simulation Monte Carlo (DSMC) method and the Boltzmann equation, the ES-BGK model is found to be able to predict accurate velocity and temperature profiles in the slip flow regime $(0.01<K n \leqslant 0.1)$, for both low-speed and high-speed flows. In the transition flow regime $(0.1<K n \leqslant 10)$, however, the model does not quantitatively capture the viscous heating effect.
\end{abstract}

(C) 2013 Elsevier Inc. All rights reserved.

\section{Introduction}

Research into non-equilibrium gas flows has been recently stimulated by the development of micro/nano-technologies, and modern material processing techniques [1,2]. The key characteristic of non-equilibrium gas flows is that the linear constitutive relations become invalid and the Navier-Stokes-Fourier (NSF) model fails. To qualitatively assess the level of nonequilibrium in the local flowfield, a commonly-used criterion is the Knudsen number $(K n)$, which is defined as the ratio of the gas mean free path to an appropriate characteristic length. The NSF equations are usually considered to be valid in the hydrodynamic flow regime where $K n \leqslant 0.01$. This validity may be extended to the slip flow regime, where $0.01<K n \leqslant 0.1$, if sufficiently accurate slip boundary conditions are applied. Flows are in the transition regime when $0.1<K n \leqslant 10$, and in the free molecular regime when $K n>10$.

The Boltzmann equation describes the dynamical behavior of a dilute gas. However, its formulation requires tracking the binary collisions of molecules. For this reason, the analysis of the Boltzmann equation, either numerically or theoretically, is practically formidable. To reduce the complexity, a number of simplified collision models have been proposed to mimic the main features of the original collision term. A simplified collision model should first satisfy conservation of mass, momentum and energy, and the Maxwellian distribution has to be achieved in equilibrium. During the collision process, which relaxes the gas towards equilibrium, the entropy production should always be positive [2]. In addition, it is desirable to have an adjustable Prandtl number (the ratio of viscosity to thermal conductivity).

The most common kinetic model may be the Bhatnagar-Gross-Krook (BGK) equation, developed in 1954 [3], where a simple relaxation term towards the Maxwellian distribution function replaces the complicated binary collision term. With

\footnotetext{
* Corresponding author. Tel.: +44 1415483773.

E-mail addresses: jianping.meng@strath.ac.uk (J. Meng), l.wu@strath.ac.uk (L. Wu), jason.reese@strath.ac.uk (J.M. Reese), yonghao.zhang@strath.ac.uk (Y. Zhang).
} 
this approximation, some accurate numerical simulations, even theoretical analysis, is possible. Most importantly, BGK predictions can be accurate for a range of practical problems. However one defect of the BGK model is that it does not recover the correct Prandtl number for monoatomic gases. Therefore, in 1966 Holway proposed the so-called ellipsoidal statistical Bhatnagar-Gross-Krook model (ES-BGK) [4], which replaced the Maxwellian equilibrium distribution with an anisotropic Guassian distribution. An additional free parameter is thereby introduced to obtain an adjustable Prantdl number. Recently, its $\mathrm{H}$-theorem has also been proved [5,6], giving the model a sound theoretical basis. In addition, the model may be extended to describe gas mixtures and gases with polyatomic molecules [4,5]. The ES-BGK model has therefore attracted considerable interest in recent years [7-10].

The accuracy of the ES-BGK model has already been investigated for a set of flow problems, e.g., one-dimensional shock structure, re-entry flow around a compression ramp and a plate, uniform shear flow, wall-bounded Couette flow and Fourier flow, and thermal creep flow [7-12,14-16,13]. Force-driven Poiseuille flow has not however been investigated. In the nearcontinuum regime, the ES-BGK model does have improved accuracy, in comparison to the BGK model, in terms of capturing thermal effects $[8,10]$. In the transition regime, it can still give better predictions for some macroscopic quantities [7-9,16], e.g., the temperature in Fourier flow, and the velocity in Couette flow. However, for shock structures, it was found that the ES-BGK model may not improve on the BGK model for large Mach numbers [11-13]. For wall-bounded Couette flows, the ESBGK may even perform worse than the BGK model in capturing the temperature profile in the transition regime: the temperature jump at bounding surfaces tends to be overestimated and the maximum at the centerline is underestimated [8]. By comparing the Sonine-polynomial coefficients for Fourier-Couette flow, it has been concluded that the molecular velocity distributions produced by the ES-BGK model are much more similar to those from the Maxwell interactions of the Boltzmann collision term, even when hard sphere interactions are actually employed [9]. For a uniform shear flow, the BGK model may give better predictions for the fourth-degree moments [15]. It is clear that our understanding of the accuracy of the ESBGK model is still incomplete and needs further studies.

To shed new light on the ES-BGK model, we investigate planar force-driven Poiseuille flow using the discrete velocity method. Our aims are twofold: assessing the model accuracy in comparison with results from the direct simulation Monte Carlo (DSMC) method [17] and the Boltzmann equation; and providing high-quality simulation data of the ES-BGK model in the slip and transition flow regimes.

\section{The ES-BGK model}

The well-known Boltzmann equation provides a complete description of a dilute monatomic gas at the molecular level. It introduces the concept of a single particle velocity distribution function $f(\boldsymbol{r}, \boldsymbol{c}, t)$ to describe the number (or portion) of molecules in the volume $d \boldsymbol{r}$ centered at position $\boldsymbol{r}=(x, y, z)$ with velocities within $d \boldsymbol{c}$ around velocity $\boldsymbol{c}=\left(c_{x}, c_{y}, c_{z}\right)$ at time $t$. Macroscopic quantities, such as the gas density $\rho$, velocity $\boldsymbol{u}$, and temperature $T$ can then be obtained as the moments of $f$, i.e.

$$
\rho[1, \boldsymbol{u}, 3 R T]=\int\left[1, \boldsymbol{c}, C^{2}\right] f d \boldsymbol{c}
$$

where $\boldsymbol{C}=\boldsymbol{c}-\boldsymbol{u}$ is the peculiar molecular velocity. Assuming that only binary collisions occur in a sufficiently dilute gas, the Boltzmann equation is:

$$
\frac{\partial f}{\partial t}+\boldsymbol{c} \cdot \frac{\partial f}{\partial \boldsymbol{r}}+\mathbf{g} \cdot \frac{\partial f}{\partial \boldsymbol{c}}=\left[\frac{\partial f}{\partial t}\right]_{\text {coll }}
$$

with

$$
\left[\frac{\partial f}{\partial t}\right]_{\text {coll }}=\int_{-\infty}^{\infty} \int_{0}^{4 \pi}\left(f^{*} f_{1}^{*}-f f_{1}\right)\left|\boldsymbol{c}-\boldsymbol{c}_{1}\right| \varrho d \Omega d \mathbf{c}_{1}
$$

where the body force $\mathbf{g}=\left(g_{x}, g_{y}, g_{z}\right)$ is assumed to be independent of the molecular velocity. In the collision term, the distributions $f$ and $f^{*}$ are evaluated at the pre-collision and post-collision molecular velocity $\boldsymbol{c}$ and $\boldsymbol{c}^{*}$, respectively. Similarly, $f_{1}$ and $f_{1}^{*}$ are evaluated for the collision pairs, and $\varrho$ is the differential collision cross section. Although more complicated multiple collisions are ignored, the collision term is still far from simple, making analysis of the Boltzmann equation difficult.

As suggested in [3], the collision term may be approximated as

$$
\left[\frac{\partial f}{\partial t}\right]_{\text {coll }}^{B G K}=\frac{1}{\tau}\left(f_{e q}-f\right),
$$

where the Maxwellian distribution $f_{e q}$ is written as

$$
f_{e q}=\rho\left(\frac{1}{2 \pi R T}\right)^{3 / 2} \exp \left[-\frac{C^{2}}{2 R T}\right]
$$


This simple BGK model makes the theoretical analysis (e.g., asymptotic analysis) and numerical simulations relatively easy. Despite its simplicity, the NSF equations can still be obtained from this model using the Chapman-Enskog technique. However, the model's main drawback is that the derived Prandtl number can only be unity. To have different Prandtl numbers, Holway [4] suggested replacing the Maxwellian distribution $f_{e q}$ function with the following anisotropic Gaussian one, i.e.

$$
\left[\frac{\partial f}{\partial t}\right]_{\text {coll }}^{E S}=\frac{1}{\tau}\left(f_{E S}-f\right)
$$

where

$$
f_{E S}=\rho \frac{1}{\sqrt{\operatorname{det}\left[2 \pi \lambda_{i j}\right]}} \exp \left[-\frac{1}{2} \lambda_{i j}^{-1} C_{i} C_{j}\right]
$$

and

$$
\lambda_{i j}=R T \delta_{i j}+b \frac{\sigma_{i j}}{\rho},
$$

where $\sigma_{i j}$ is the stress. As the matrix $\lambda_{i j}^{-1}$ must be positive definite, the parameter $b$ is restricted to $-\frac{1}{2} \leqslant b \leqslant 1$. Using the Chapman-Enskog technique, the viscosity and thermal conductivity can be derived as

$$
\mu=\frac{1}{1-b} p \tau, \quad \kappa=\frac{5}{2} p R \tau
$$

Therefore, the Prandtl number is $\operatorname{Pr}=1 /(1-b)$, which is adjustable via the free parameter $b$. The correct Prandtl number of an ideal gas, $\operatorname{Pr}=2 / 3$, can be recovered with $b=-1 / 2$. When $b=0$ the ES-BGK model reduces to the BGK model.

In addition, Shakhov also proposed a so-called S-model to fix the Prandtl number issue [18,19], where the "collision term" can be written as

$$
\left[\frac{\partial f}{\partial t}\right]_{\text {coll }}^{S}=\frac{1}{\tau}\left(f_{S}-f\right)
$$

where

$$
f_{S}=f_{e q}\left[1+\frac{1-\operatorname{Pr}}{5} \frac{2 q_{i} C_{i}}{p R T}\left(\frac{C^{2}}{2 R T}-\frac{5}{2}\right)\right]
$$

and $q_{i}$ is the heat flux.

\section{Numerical scheme}

As with the BGK model [20], for a spatially one-dimensional problem we can eliminate gas molecular speeds $c_{x}$ and $c_{z}$ in the simulations (assuming that $c_{y}$ is perpendicular to the walls). It is convenient to introduce the following dimensionless variables,

$$
\begin{aligned}
& \hat{x}_{k}=\frac{x_{k}}{L}, \quad \hat{u}_{k}=\frac{u_{k}}{\sqrt{R T_{0}}}, \quad \hat{t}=\frac{\sqrt{R T_{0}} t}{L}, \quad \hat{g}_{k}=\frac{L g_{k}}{R T_{0}}, \quad \hat{c}_{k}=\frac{c_{k}}{\sqrt{R T_{0}}}, \quad \hat{T}=\frac{T}{T_{0}}, \\
& \hat{f}=\frac{f\left(R T_{0}\right)^{3 / 2}}{\rho_{0}}, \quad \hat{\rho}=\frac{\rho}{\rho_{0}}, \quad \hat{p}=\frac{p}{p_{0}}, \quad \hat{\mu}=\frac{\mu}{\mu_{0}}, \quad \hat{q}_{i}=\frac{q_{i}}{p_{0} \sqrt{R T_{0}}}, \quad \hat{P}_{i j}=\frac{P_{i j}}{p_{0}} .
\end{aligned}
$$

The governing equation can then be rewritten as

$$
\frac{\partial \hat{f}}{\partial \hat{t}}+\hat{c}_{k} \frac{\partial \hat{f}}{\partial \hat{x}_{k}}+\hat{g}_{k} \frac{\partial \hat{f}}{\partial \hat{c}_{k}}=-\operatorname{Pr} \frac{p_{0} L}{\mu_{0} \sqrt{R T_{0}}} \frac{\hat{p}}{\hat{\mu}}\left(\hat{f}-\hat{f}_{E S}\right)=-\operatorname{Pr} \frac{\hat{\rho} \hat{T}^{(1-\omega)}}{K n}\left(\hat{f}-\hat{f}_{E S}\right)
$$

where the Knudsen number is defined as

$$
K n=\frac{\mu_{0} \sqrt{R T_{0}}}{p_{0} L} .
$$

The relevant macroscopic quantities are

$$
\left[\begin{array}{c}
\hat{\rho} \\
\hat{\rho} \hat{u}_{i} \\
\hat{P}_{i j} \\
3 \hat{\rho} \hat{T}
\end{array}\right]=\int \hat{f}\left[\begin{array}{c}
1 \\
\hat{C}_{i} \\
\hat{C}_{i} \hat{C}_{j} \\
\hat{C}_{i} \hat{C}_{i}
\end{array}\right] d \hat{\boldsymbol{c}} .
$$


In thermal flows the viscosity depends on temperature, which can be expressed as $\mu / \mu_{0}=\left(T / T_{0}\right)^{\omega}$, where $\omega$ is related to the molecular interaction model and varies from 0.5 for hard-sphere molecular interactions to 1 for Maxwell molecules. The hat symbol will be omitted hereafter for clarity. A rescaled Knudsen number,

$$
K_{D}=\sqrt{\frac{\pi}{2}} K n
$$

is also used throughout this work for convenient comparison with the DSMC data. ${ }^{1}$ When we refer below to the Knudsen number, it should be understood as $K_{D}$.

To solve a spatially one-dimensional problem such as Couette, or force-driven Poiseuille, or Fourier flows, we can introduce the following marginal velocity distribution functions, and the corresponding parts for the anisotropic Gaussian distribution:

$$
\begin{aligned}
& {\left[\begin{array}{l}
\varphi_{a} \\
\varphi_{b} \\
\varphi_{c} \\
\varphi_{d}
\end{array}\right]=\int_{-\infty}^{\infty} \int_{-\infty}^{\infty}\left[\begin{array}{l}
1 \\
c_{x} \\
c_{x}^{2} \\
c_{z}^{2}
\end{array}\right] f d c_{x} d c_{z},} \\
& {\left[\begin{array}{l}
\varphi_{a e} \\
\varphi_{b e} \\
\varphi_{c e} \\
\varphi_{d e}
\end{array}\right]=\int_{-\infty}^{\infty} \int_{-\infty}^{\infty}\left[\begin{array}{c}
1 \\
c_{x} \\
c_{x}^{2} \\
c_{z}^{2}
\end{array}\right] f_{E S} d c_{x} d c_{z}=\frac{\rho e^{-\frac{c_{y}^{2}}{2 \lambda y y}}}{\sqrt{2 \pi \lambda_{\mathrm{yy}}}}\left[\begin{array}{c}
1 \\
\frac{2 u_{x} c_{y} \lambda_{\mathrm{xy}}}{\lambda_{\mathrm{yy}}}+\frac{c_{y} \lambda_{\mathrm{xy}} \lambda_{\mathrm{xy}}^{2}}{\lambda_{\mathrm{yy}}^{2}}+u_{x}^{2}+\lambda_{\mathrm{xx}}-\frac{\lambda_{\mathrm{xy}}^{2}}{\lambda_{\mathrm{yy}}} \\
\lambda_{\mathrm{zz}}
\end{array}\right] .}
\end{aligned}
$$

With these marginal distribution functions, the macroscopic quantities in Eq. (6) become

$$
\left[\begin{array}{c}
\rho \\
\rho u_{x} \\
3 \rho T \\
P_{x x} \\
P_{x y} \\
P_{y y} \\
P_{z z}
\end{array}\right]=\int_{-\infty}^{\infty}\left[\begin{array}{c}
\varphi_{a} \\
\varphi_{b} \\
\varphi_{c}+\varphi_{d}+c_{y}^{2} \varphi_{a} \\
\varphi_{c} \\
\varphi_{b} c_{y}-u_{x} \varphi_{a} c_{y} \\
c_{y}^{2} \varphi_{a} \\
\varphi_{d}
\end{array}\right] d c_{y}+\left[\begin{array}{c}
0 \\
0 \\
-\rho u_{x}^{2} \\
-\rho u_{x}^{2} \\
0 \\
0 \\
0
\end{array}\right] .
$$

Now let

$$
\phi=\left[\begin{array}{c}
\varphi_{a} \\
\varphi_{b} \\
\varphi_{c} \\
\varphi_{d}
\end{array}\right], \quad \phi_{e}=\left[\begin{array}{c}
\varphi_{a e} \\
\varphi_{b e} \\
\varphi_{c e} \\
\varphi_{d e}
\end{array}\right], \quad S=g_{x}\left[\begin{array}{c}
0 \\
\varphi_{a} \\
2 \varphi_{b} \\
0
\end{array}\right],
$$

so the governing equation for the four distribution functions can be written as

$$
\frac{\partial \phi}{\partial t}+c_{y} \frac{\partial \phi}{\partial y}=\frac{\operatorname{Pr}}{K n} \rho T^{(1-\omega)}\left(\phi_{e}-\phi\right)+S
$$

In particular, if the problem is steady, Eq. (9) can be further reduced to

$$
c_{y} \frac{\partial \phi}{\partial y}=\frac{\operatorname{Pr}}{K n} \rho T^{(1-\omega)}\left(\phi_{e}-\phi\right)+S
$$

where the time variable is eliminated. In these equations the corresponding differential force terms have been transformed into non-differential source term $S$ by utilizing integration by parts. For example,

$$
\int_{-\infty}^{\infty} \int_{-\infty}^{\infty} c_{x} g_{x} \frac{\partial f}{\partial c_{x}} d c_{x} d c_{z}=g_{x} \int_{-\infty}^{\infty} \int_{-\infty}^{\infty}\left(\frac{\partial c_{x} f}{\partial c_{x}}-f\right) d c_{x} d c_{z}=g_{x}\left[\int\left(\left.f c_{x}\right|_{c_{x}=-\infty} ^{c_{x}=\infty}\right) d c_{z}-\varphi_{a}\right]=-g_{x} \varphi_{a}
$$

As $f$ is assumed to be decaying sufficiently fast, its product with power functions of $\boldsymbol{c}$ is zero when the components of $\boldsymbol{c}$ approach infinity. The force terms can be obtained similarly for other marginal distribution functions.

\footnotetext{
1 There are different non-dimensional systems. For example, the reference velocity can be chosen as $\sqrt{2 R T_{0}}$, which leads to a factor of $\sqrt{2}$ difference from the present non-dimensional velocity. Also, there can be different formulations for the Knudsen number, although one can easily transform between them if necessary. For example, if the mean free path is calculated as $\frac{\sqrt{\pi} \mu}{2 p_{0}} \sqrt{2 R T_{0}}$, one can identify the relation with the so-called rarefaction parameter $\delta$ [21], i.e. $\sqrt{2 / \pi} K_{D}=K n=1 / \sqrt{2} \delta$.
} 
Once the above macroscopic quantities are determined by solving Eq. (9) or (10), other high order moments like heat flux may be evaluated by introducing additional marginal distribution functions [20].

As a fully-developed problem is studied here, Eq. (10) needs to be solved numerically. For this purpose, we need to discretize in a two-dimensional space, i.e. one-dimension in the physical space $y$, and one-dimension in the molecular velocity space $c_{y}$. For the molecular velocity space, Simpson's rule [22] is chosen for $c_{y}$, and the grid points are distributed uniformly. For the physical space, nonuniform grid points are employed with more points near the boundary. To construct this kind of grid, we first obtain a distribution of points which is highly dense near the middle point of the channel by using

$$
y_{i}=\alpha \sinh \left[\sinh ^{-1}\left(\frac{1}{2 \alpha}\right) \frac{i}{N}+\sinh ^{-1}\left(\frac{-1}{2 \alpha}\right)\left(1-\frac{i}{N}\right)\right], \quad i=0 \ldots N,
$$

where $N$ denotes the total number of points and $\alpha$ is the parameter determining the nonuniformity. Then, the grid system can be made to be denser near the wall by utilizing symmetry and translation relations.

Regarding the numerical scheme, we employ a second-order upwind scheme, except in the near-wall region where a first-order upwind scheme is used. Therefore, the evolution of $\phi$ can be written as

$$
\phi_{i}=\frac{c_{y}\left(\eta_{i}^{2} \phi_{i-1}-\phi_{i-2}\right)+d y_{i} \eta_{i}\left(\eta_{i}-1\right)\left(w_{i} \phi_{e, i}+S_{i}\right)}{\left(\eta_{i}-1\right)\left(\eta_{i} c_{y}+c_{y}+d y_{i} \eta_{i} w_{i}\right)}, \quad c_{y}>0, \quad i=2 \ldots N
$$

and

$$
\phi_{1}=\frac{c_{y} \phi_{0}+d y_{1} S_{1}+d y_{1} w_{1} \phi_{e, 1}}{c_{y}+d y w_{1}}, \quad c_{y}>0
$$

where

$$
\begin{aligned}
& w_{i}=\frac{\operatorname{Pr} \rho_{i} T_{i}^{1-\omega}}{K n}, \\
& d y_{i}=y_{i}-y_{i-1}, \quad i=1 \ldots N
\end{aligned}
$$

and

$$
\eta_{i}=\frac{d y_{i}+d y_{i-1}}{d y_{i}}, \quad i=2 \ldots N
$$

For simplicity, the rules for $c_{y}<0$ are omitted here; they can be easily obtained in a manner similar to the above.

In our simulations, a diffuse kinetic boundary condition is used, which can be written as follows:

$$
f\left(y= \pm \frac{1}{2}, \pm c_{y}<0\right)=\frac{\rho_{w}}{\left(2 \pi T_{w}\right)^{3 / 2}} \exp \left(-\frac{C_{w}^{2}}{2 T_{w}}\right)
$$

with

$$
\rho_{w}=\sqrt{\frac{2 \pi}{T_{w}}} \int_{ \pm c_{y}>0}\left|c_{y} f\left(y= \pm \frac{1}{2}, \pm c_{y}>0\right)\right| d \boldsymbol{c} .
$$

When using the above four marginal distribution functions, this can be transformed to

$$
\left[\begin{array}{c}
\varphi_{a w} \\
\varphi_{b w} \\
\varphi_{c w} \\
\varphi_{d w}
\end{array}\right]=\int_{-\infty}^{\infty} \int_{-\infty}^{\infty}\left[\begin{array}{c}
1 \\
c_{x} \\
c_{x}^{2} \\
c_{z}^{2}
\end{array}\right] f_{e q, w} d c_{x} d c_{z}=\frac{\rho_{w} \exp \left(-\frac{c_{y}^{2}}{2 T_{w}}\right)}{\sqrt{2 \pi T_{w}}}\left[\begin{array}{c}
1 \\
u_{w} \\
T_{w}+u_{w}^{2} \\
T_{w}
\end{array}\right],
$$

where the density $\rho_{w}$ becomes,

$$
\rho_{w}=\sqrt{\frac{2 \pi}{T_{w}}} \int_{ \pm c_{y}>0}\left|\varphi_{a}\left( \pm \frac{1}{2}\right) c_{y}\right| d c_{y} .
$$

\section{Simulation results}

We evaluate the ES-BGK model for force-driven Poiseuille flows, where the gas is confined between two parallel infinite plates located at $y=0$ and $y=1$ in the nondimensional system. Both plates are at rest and their temperature is maintained at $T_{w}=1$. The gas is subject to a uniform external force in the $x$ direction, i.e. the flow direction is parallel to the plates. Due to the presence of this force, this Poiseuille flow is more interesting than Couette or Fourier flows, as it presents a bi-modal temperature profile that the NSF equations fail to predict even qualitatively [20,23-26]. To assess the modeling accuracy of 
the ES-BGK equation, $g_{x}=0.22$ and 1 (which are also close to the values used in previous BGK simulations [20] and DSMC simulations [23]) are chosen in the following simulations. We focus on fully-developed flows, and the diffuse kinetic boundary condition is employed for the gas-wall interactions.

To achieve satisfactory accuracy, it is important to choose appropriate molecular velocity and spatial grids. For this purpose, each simulation is run on two discretized systems with the finer one usually having double the molecular velocity and spatial points. Only when the difference between the results for these two systems is sufficiently small do we consider the coarser grid acceptable. The difference is evaluated based on two types of errors: the first type, $\epsilon^{1}$, is the relative difference in the macroscopic quantities at the chosen points in the two systems, i.e.

$$
\epsilon_{i}^{1}=\left|\frac{Q_{i}^{\complement}-Q_{i}^{\mathcal{F}}}{Q_{i}^{\mathcal{F}}}\right|,
$$

where $Q$ denotes the macroscopic quantities to be evaluated. The superscript $\complement$ represents values obtained on the coarser grid and $\mathcal{F}$ on the finer grid, and $i$ is the point index. The second error, $\epsilon^{2}$, is the difference in the macroscopic quantities in the two systems relative to the maximum difference in the corresponding macroscopic quantities in the finer system, i.e.

$$
\epsilon_{i}^{2}=\left|\frac{Q_{i}^{\complement}-Q_{i}^{\mathcal{F}}}{\max \left(Q^{\mathcal{F}}\right)-\min \left(Q^{\mathcal{F}}\right)}\right|
$$

Specifically, we choose the macroscopic velocity and temperature as the benchmark quantities. The two errors are evaluated at the six points $[0,0.01,0.1,0.2,0.3,0.4,0.5]$ which include the boundary point. As the present problem setup is symmetrical, these points effectively cover the full space. If both types of errors for temperature and velocity in the coarser system are less than $1 \%$ in comparison with the finer system, we accept the coarser system to be sufficient. According to these criteria, we determine an appropriate discretized system for each set of parameters in the following simulations. The typical spatial grid point number is 200 with which we obtain a minimal grid size of 0.000461 near the boundary. For the molecular velocity, the typical number of grid points is 10,000 . Nevertheless, either grid point number may be increased to achieve satisfactory accuracy. For instance a finer molecular velocity grid becomes necessary for Knudsen numbers $K_{D} \geqslant 3$, particularly for $K_{D}=10$. As the temperature variation, i.e. $\max (T)-\min (T)$, can be small for some Knudsen numbers (e.g., $K_{D}=0.4$ and 0.5 ), $\epsilon^{2}$ becomes sensitive so that a finer spatial grid ( 400 points) is also necessary.

To further verify the numerical implementation, two cases with $g_{x}=1$ are run using the Maxwell interaction model with $\operatorname{Pr}=1$, i.e. $b$ is set to be 0 and $\omega$ is 1 . These simulation results should be directly comparable with the BGK data reported in [20], and both are presented in Fig. 1. The agreement confirms the validity of our chosen grids for both molecular velocity and space.

Another issue is the truncation of the molecular velocity space. Although this space is infinite, appropriate bounds have to be chosen for practical simulations. These may be estimated by a combination of the maximum of the macroscopic velocity and temperature, i.e. $\max \left(u_{x}\right)+\max (a \sqrt{T})$ [8]. Here, $a$ is set to be 12 , so the bound is chosen to be [-20,20]. The validity of this velocity bound is also confirmed by a comparison between the bounds $[-20,20]$ and $[-30,30]$ for the case $K_{D}=0.05$ which is shown in Fig. 2. The errors for both velocity and temperature effectively approach zero (less than $\left.O\left(10^{-11}\right)\right)$. As the maximum velocity and temperature for the low Knudsen number cases are usually larger than those in the considered high Knudsen number cases, this bound is employed for all simulations.

Figs. 2-4 show comparisons between the results of the ES-BGK model and the DSMC particle technique for $K_{D}=0.05,0.1,1$ and 5 , and two body forces $g_{x}=0.22,1$. When $K_{D}=0.05$, the ES-BGK model agrees with the DSMC, but

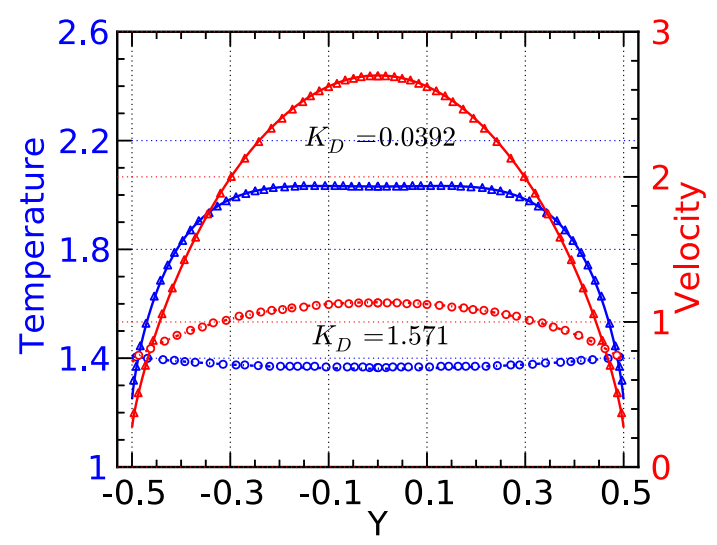

Fig. 1. Convergence analysis: the present results (lines) are compared with the BGK data (symbols) reported by Aoki et al. [20]. Maxwell gas interactions are used in both models, and the results are comparable by letting $b=0, \omega=1$ in the ES-BGK model. Two Knudsen numbers 0.0392 (the upper lines and symbols) and 1.571 (the lower lines and symbols) are considered. The agreement between the two set of results confirms the validity of our chosen molecular velocity and spatial grids. 

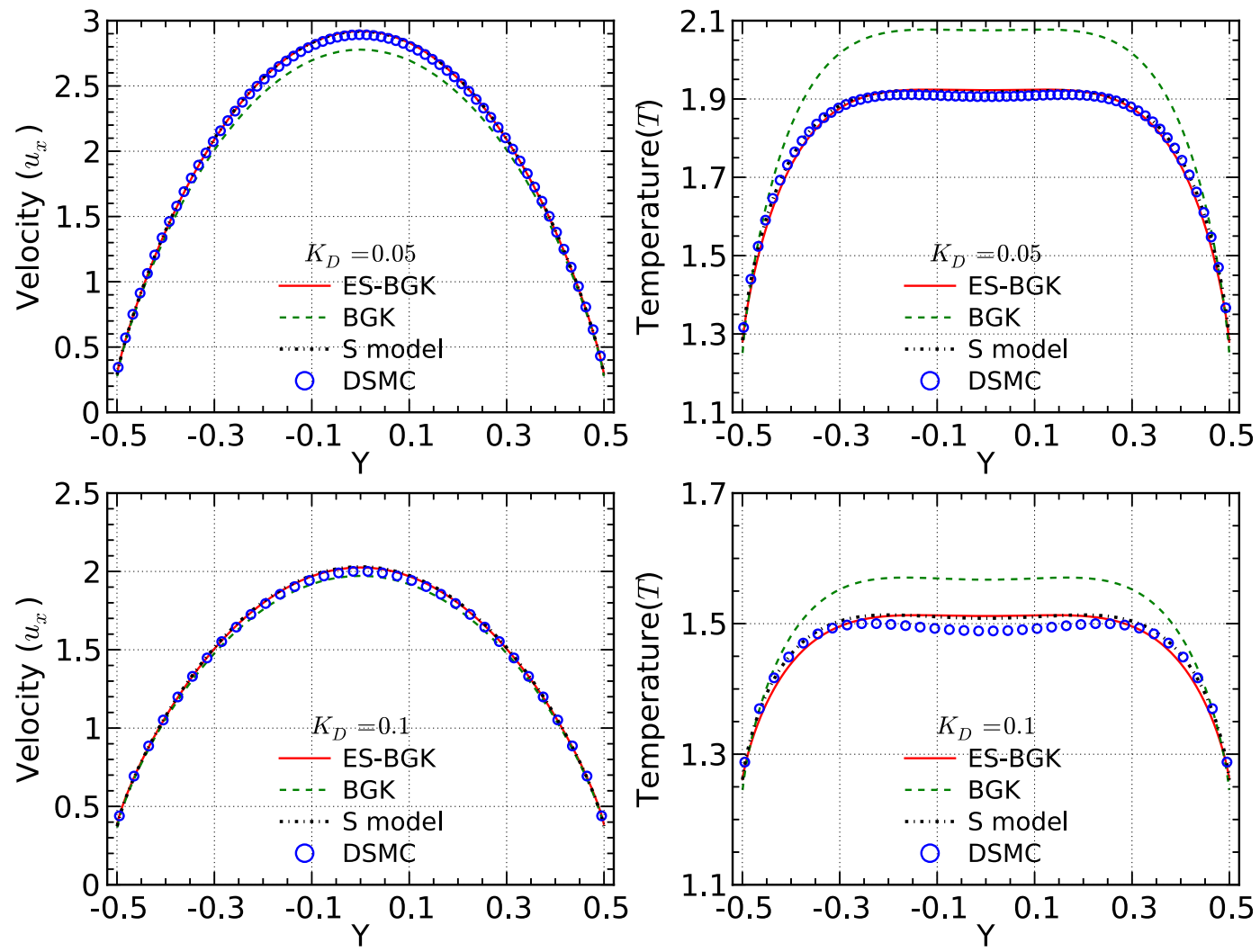

Fig. 2. Velocity and temperature profiles of force-driven Poiseuille flows for $K_{D}=0.05,0.1$ with $g_{x}=1$. Hard sphere molecular interactions are considered, i.e. $\omega=0.5$. The solid lines are the ES-BGK data with $\operatorname{Pr}=2 / 3$ and the symbols are the DSMC data. The BGK data (dashed lines) and the S-model data (dashed-dotted lines) are also included.
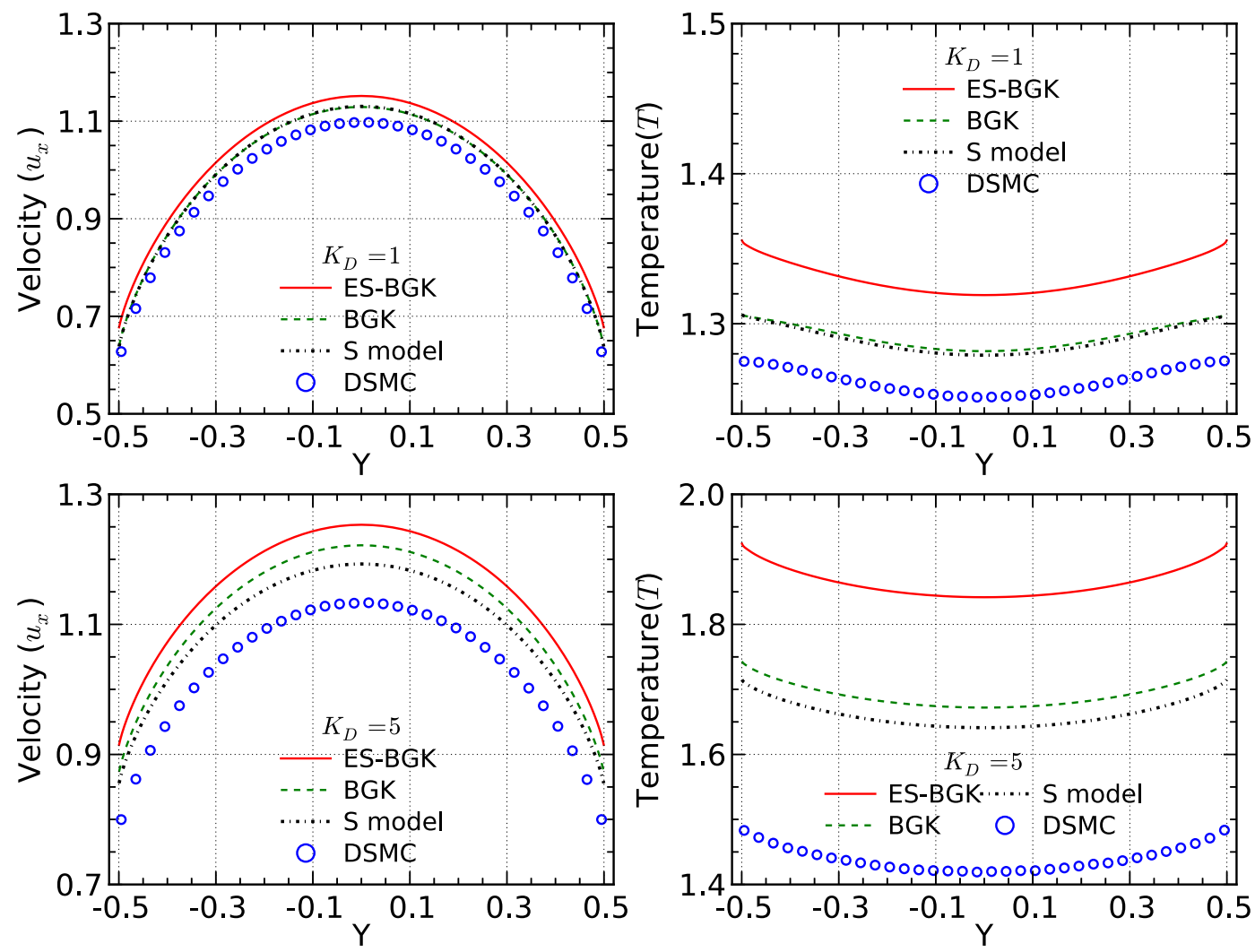

Fig. 3. Velocity and temperature profiles of force-driven Poiseuille flows for $K_{D}=1,5$ with $g_{x}=1$. Hard sphere molecular interactions are considered, i.e. $\omega=0.5$. The solid lines are the ES-BGK data with $P r=2 / 3$ and the symbols are the DSMC data. The BGK data (dashed lines) and the S-model data (dasheddotted lines) are also included. 

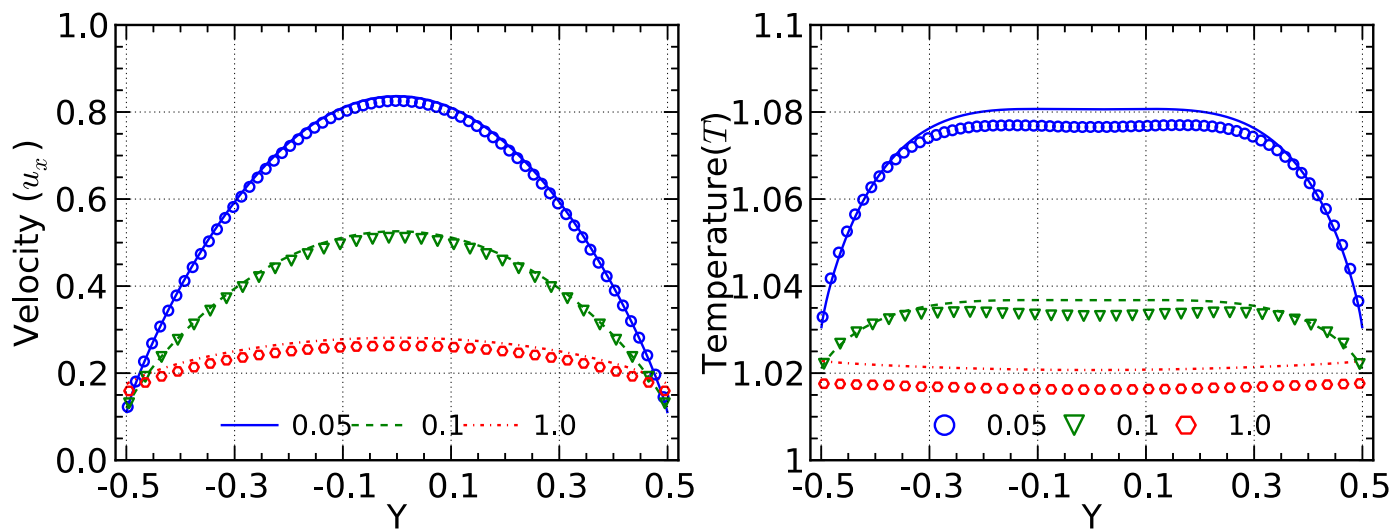

Fig. 4. Velocity and temperature profiles of force-driven Poiseuille flows with $g_{x}=0.22$. Hard sphere molecular interactions are considered, i.e. $\omega=0.5$. The lines are the ES-BGK data with $P r=2 / 3$ and the symbols are the DSMC data. The Knudsen numbers are $K_{D}=0.05,0.1$ and 1.0 as noted and they are distinguished by different plot styles as shown.
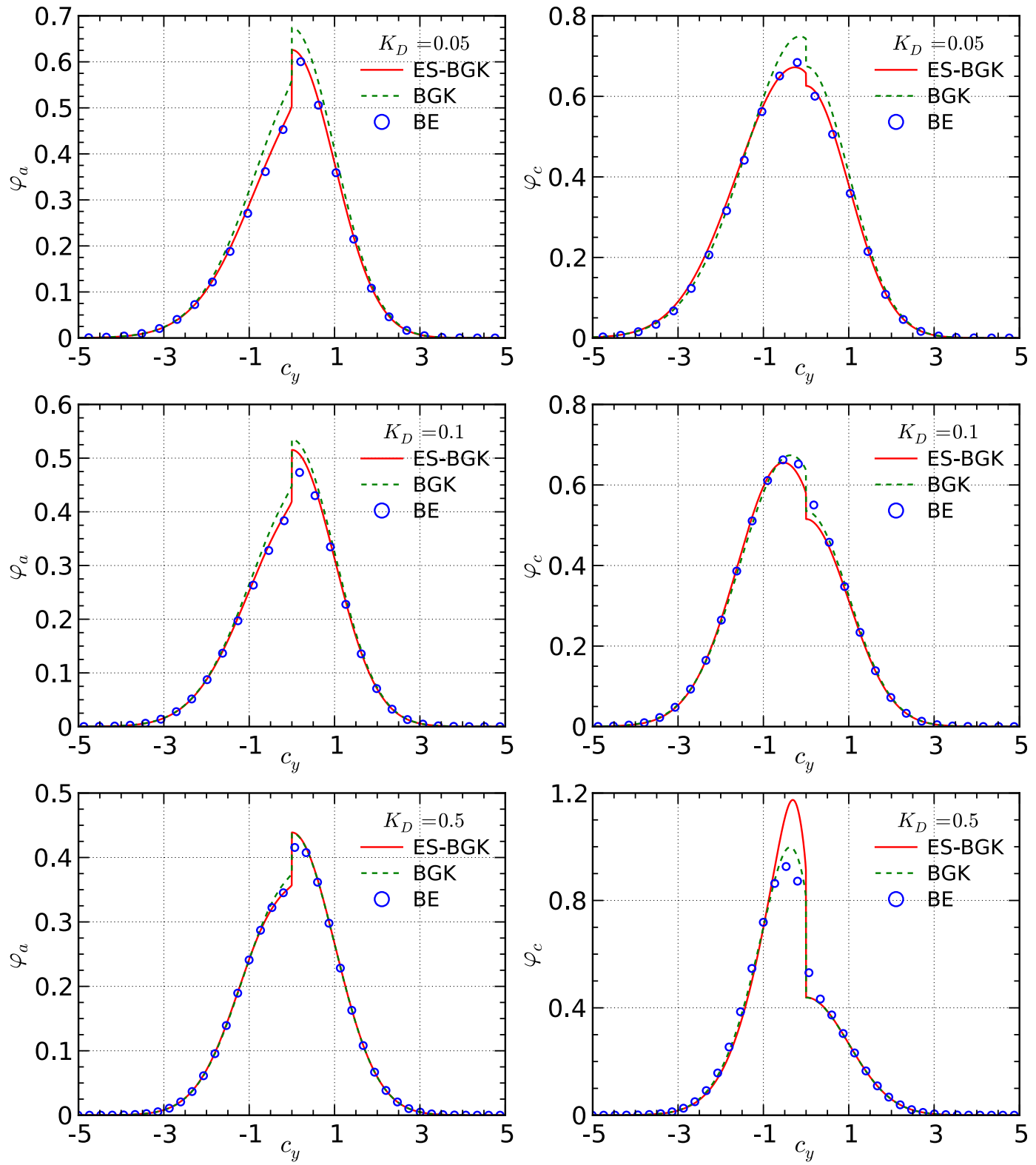

Fig. 5. Comparisons of BGK and ES-BGK marginal distribution functions $\varphi_{a}$ and $\varphi_{c}$ at the left wall boundary ( $\left.y=-0.5\right)$ with those of the Boltzmann equation $(\mathrm{BE})$ for $g_{x}=1$. 
as $K_{D}$ increases to 0.1 , the deviation from the DSMC data becomes larger although the agreement is still satisfactory. Specifically, however, the viscous heating effect is not accurately captured; although the phenomenon of bi-modal temperature distribution is captured qualitatively, the bimodality is not as significant as the DSMC prediction. When $K_{D}$ increases to 1.0, the ES-BGK model tends to overestimate the temperature profile for this force-driven flow, while it underestimates it for Couette flow in the transition flow regime [8].

It is informative to compare the ES-BGK model with the BGK model and the S-model. For lower Knudsen numbers, both the ES-BGK model and the $S$ model predict more realistic temperature profiles than the BGK model. This should be attributed to the fact that the Prandtl number issue is corrected. However, for larger Knudsen numbers such as $K_{D}=1\left(g_{x}=0.22\right.$ and $\left.g_{x}=1\right)$ and $5\left(g_{x}=1\right)$, the temperature predictions become unsatisfactory for all three models. For $g_{x}=1$ velocity predictions also show large errors in comparison to the DSMC results, although they appear to be acceptable for $g_{x}=0.22$ and $K_{D}=1$ where the viscous heating effect is less significant.

The ES-BGK model predicts the highest temperature among the three models for $K_{D}=1$ and 5 with $g_{x}=1$. These predictions are even worse than the BGK model, which is the opposite of the situation at small Knudsen number. Meanwhile, the accuracy of the $\mathrm{S}$ model appears to be at least no worse than the BGK model.

To further investigate this phenomenon, we compare the marginal distribution functions (see Eq. (7)) with direct solutions of the full Boltzmann equation using the numerical method reported in [27,28], where the molecular velocity distribution function can be obtained accurately. The results for $\varphi_{a}$ and $\varphi_{c}$ at the left wall boundary and the middle channel point are shown in Figs. 5 and 6.
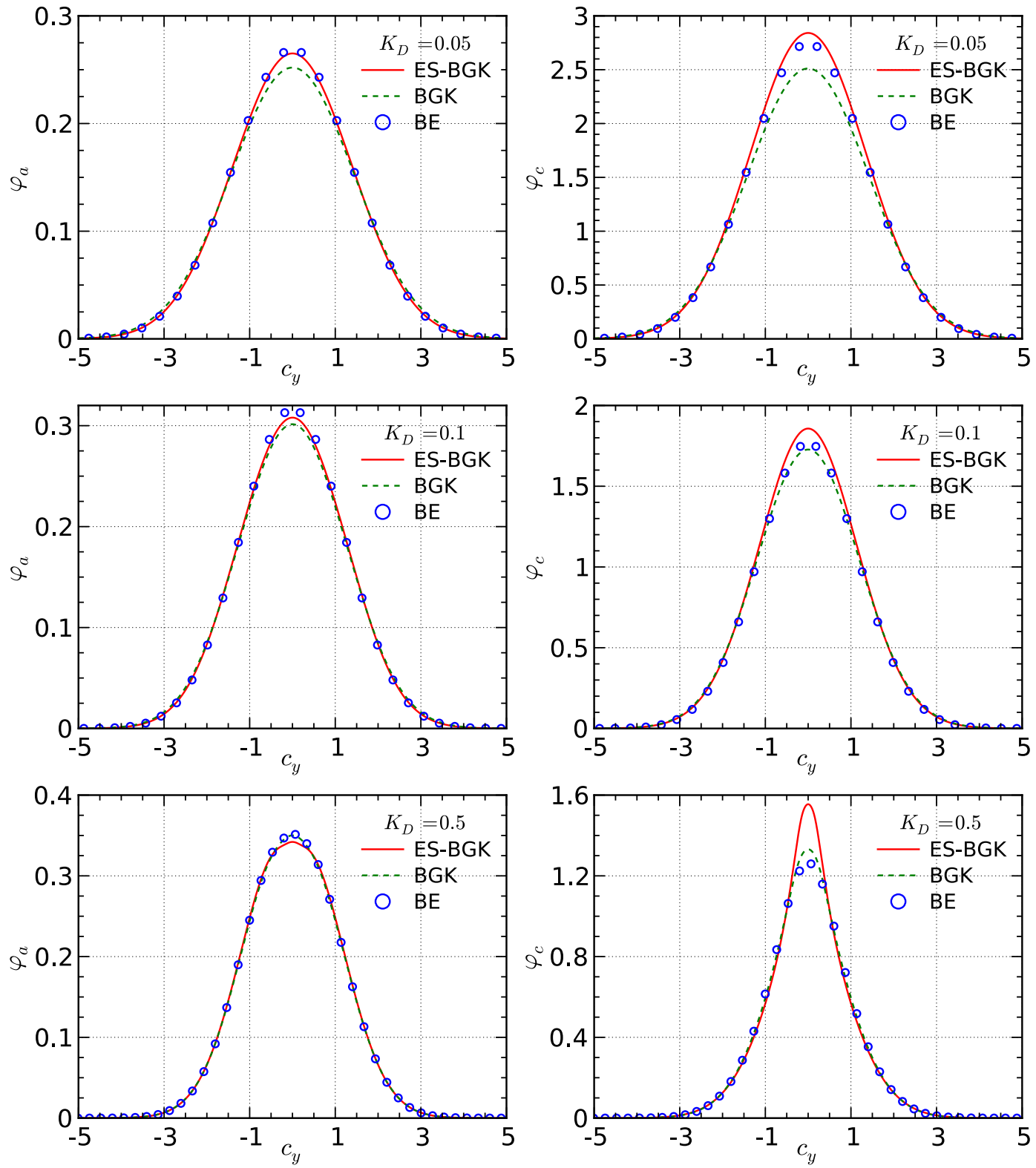

Fig. 6. Comparisons of BGK and ES-BGK marginal distribution functions $\varphi_{a}$ and $\varphi_{c}$ at the middle point $(y=0)$ with those of the Boltzmann equation (BE) for $g_{x}=1$. 

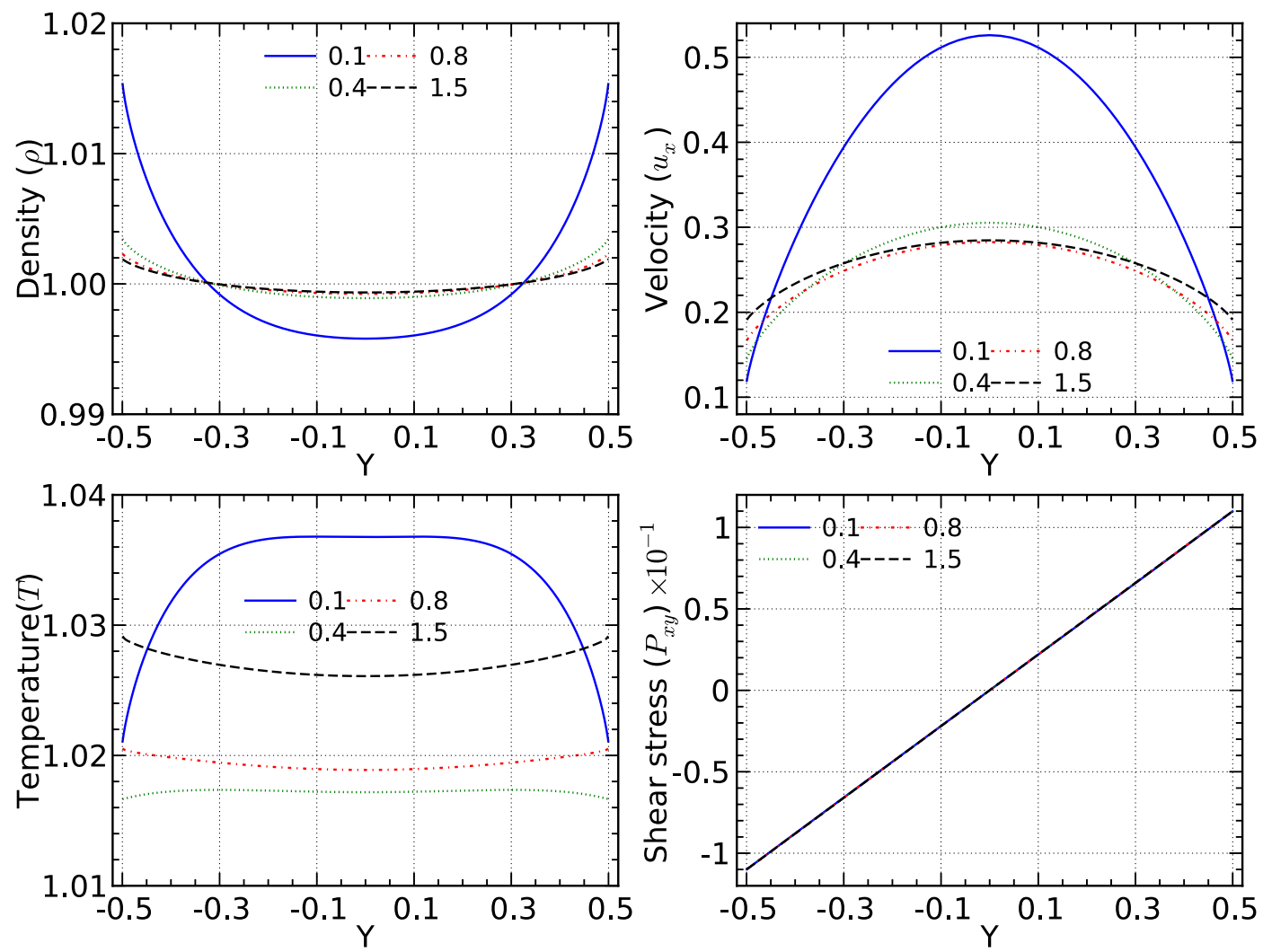

Fig. 7. Simulation results of the ES-BGK model applied to force-driven Poiseuille flow at various Knudsen numbers (labeled) and for $g_{x}=0.22$.
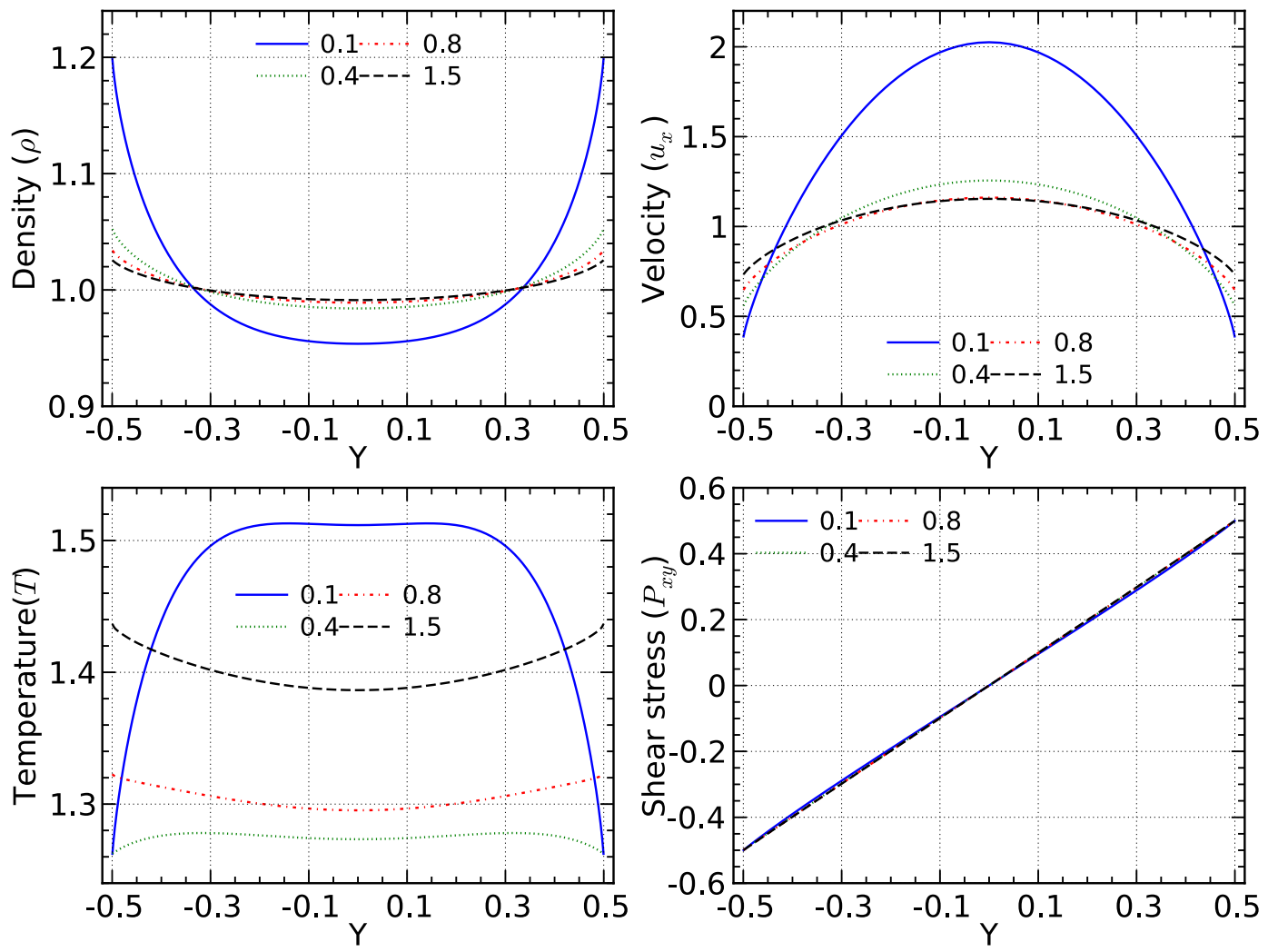

Fig. 8. Simulation results of the ES-BGK model applied to force-driven Poiseuille flow at for various Knudsen numbers (labeled) and for $g_{x}=1$. 
It is evident that the ES-BGK model can provide more accurate distribution functions when the Knudsen number is smaller than 0.1. However, for Knudsen numbers larger than 0.1, the ES-BGK equation tends to produce less accurate distribution functions. When $K_{D}$ is $0.5, \varphi_{c}$ is even quantitatively worse than the one predicted by the BGK model. This is directly responsible for the worse performance of the ES-BGK model in predicting the temperature-field at larger Knudsen numbers.

As kinetic models are approximations to the Boltzmann equation in which the particle interactions are simplified, they should not be expected to be particularly accurate if the details of the collisions play a vital role, as for viscous heating effects. As the temperature rise greatly influences the gas properties such as the viscosity, the predictions of other macroscopic quantities may then not be sufficiently accurate for high Mach number flows at larger Knudsen numbers. However, when the body force is relatively small, the temperature rise may be insignificant, and then reasonable predictions for other macroscopic quantities could be given by the ES-BGK model as the gas properties will not change too much. For instance, the velocity profiles in Fig. 4 are in satisfactory agreement with those of the DSMC simulations. This echoes the previous findings of satisfactory predictions of velocity profiles for Couette flow when the Mach number is not very large [9,8]. In particular, when viscous heating is negligible, even the temperature field may be predicted accurately, e.g., Fourier flows [9,7].

More simulation data for the force-driven flow are presented in Figs. 7 and 8. In the near-continuum regime, the temperature profiles are parabola-like and open downward. They become bimodal for intermediate Knudsen numbers, and then return to a parabola-like shape again but open upward for larger Knudsen numbers. The density and velocity profiles maintain a parabola-like shape. By contrast, the shear stress has a nearly linear-like profile except in the cases of $g_{x}=1$.

The mass and heat flow rates,

$$
\mathcal{M}=\int_{-1 / 2}^{1 / 2} \rho u_{x} d y, \quad \mathcal{H}=\int_{-1 / 2}^{1 / 2}\left(\int \frac{1}{2} C_{x} C^{2} f d c\right) d y
$$
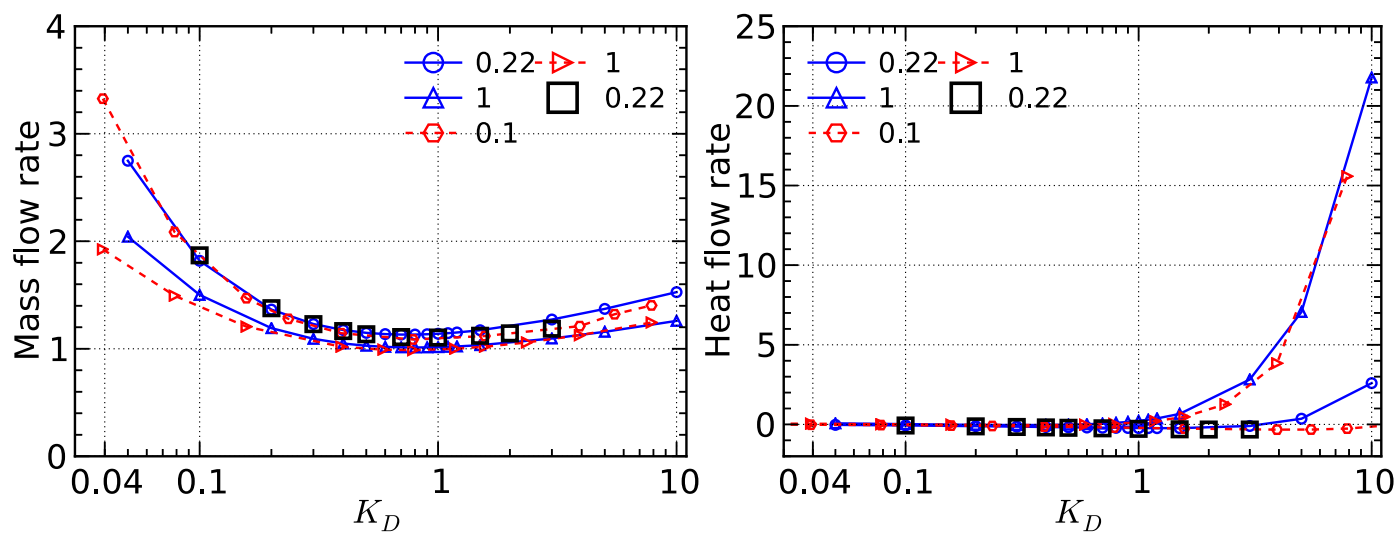

Fig. 9. Mass and heat flow rates predicted by the ES-BGK model (the solid lines with circles and upper triangles), the Boltzmann equation (the squares) and the BGK model (the dashed lines with hexagons and right triangles). The body force magnitudes are presented in the legend. The BGK data are from Table 2 of Aoki et al. [20], with the Knudsen numbers converted to $K_{D}$ accordingly. The mass and heat flow rates are further normalized by the corresponding body force magnitude.

Table 1

Non-dimensional mass flow $(\mathcal{M})$ and heat flow $(\mathcal{H})$ rates predicted by the ES-BGK model.

\begin{tabular}{|c|c|c|c|c|}
\hline \multirow[t]{2}{*}{$K_{D}$} & \multicolumn{2}{|l|}{$\mathcal{M}$} & \multicolumn{2}{|l|}{$\mathcal{H}$} \\
\hline & $g_{x}=0.22$ & $g_{x}=1$ & $g_{x}=0.22$ & $g_{x}=1$ \\
\hline 0.05 & $6.047 \times 10^{-1}$ & $2.042 \times 10^{0}$ & $-6.225 \times 10^{-3}$ & $6.864 \times 10^{-2}$ \\
\hline 0.1 & $4.000 \times 10^{-1}$ & $1.501 \times 10^{0}$ & $-1.408 \times 10^{-2}$ & $1.892 \times 10^{-2}$ \\
\hline 0.2 & $3.002 \times 10^{-1}$ & $1.192 \times 10^{0}$ & $-2.402 \times 10^{-2}$ & $-2.647 \times 10^{-2}$ \\
\hline 0.3 & $2.706 \times 10^{-1}$ & $1.092 \times 10^{0}$ & $-3.073 \times 10^{-2}$ & $-3.958 \times 10^{-2}$ \\
\hline 0.4 & $2.583 \times 10^{-1}$ & $1.049 \times 10^{0}$ & $-3.574 \times 10^{-2}$ & $-3.496 \times 10^{-2}$ \\
\hline 0.5 & $2.526 \times 10^{-1}$ & $1.028 \times 10^{0}$ & $-3.966 \times 10^{-2}$ & $-1.697 \times 10^{-2}$ \\
\hline 0.6 & $2.500 \times 10^{-1}$ & $1.018 \times 10^{0}$ & $-4.279 \times 10^{-2}$ & $1.217 \times 10^{-2}$ \\
\hline 0.7 & $2.491 \times 10^{-1}$ & $1.013 \times 10^{0}$ & $-4.530 \times 10^{-2}$ & $5.152 \times 10^{-2}$ \\
\hline 0.8 & $2.491 \times 10^{-1}$ & $1.012 \times 10^{0}$ & $-4.730 \times 10^{-2}$ & $1.002 \times 10^{-1}$ \\
\hline 0.9 & $2.497 \times 10^{-1}$ & $1.013 \times 10^{0}$ & $-4.886 \times 10^{-2}$ & $1.576 \times 10^{-1}$ \\
\hline 1.0 & $2.507 \times 10^{-1}$ & $1.015 \times 10^{0}$ & $-5.005 \times 10^{-2}$ & $2.231 \times 10^{-1}$ \\
\hline 1.1 & $2.520 \times 10^{-1}$ & $1.018 \times 10^{0}$ & $-5.089 \times 10^{-2}$ & $2.965 \times 10^{-1}$ \\
\hline 1.2 & $2.533 \times 10^{-1}$ & $1.021 \times 10^{0}$ & $-5.141 \times 10^{-2}$ & $3.773 \times 10^{-1}$ \\
\hline 1.5 & $2.579 \times 10^{-1}$ & $1.034 \times 10^{0}$ & $-5.133 \times 10^{-2}$ & $6.609 \times 10^{-1}$ \\
\hline 3.0 & $2.798 \times 10^{-1}$ & $1.095 \times 10^{0}$ & $-2.151 \times 10^{-2}$ & $2.820 \times 10^{0}$ \\
\hline 5.0 & $3.016 \times 10^{-1}$ & $1.158 \times 10^{0}$ & $7.863 \times 10^{-2}$ & $7.061 \times 10^{0}$ \\
\hline 10.0 & $3.359 \times 10^{-1}$ & $1.259 \times 10^{0}$ & $5.704 \times 10^{-1}$ & $2.178 \times 10^{1}$ \\
\hline
\end{tabular}


respectively, predicted by the ES-BGK model are shown in Fig. 9 and in Table 1. The BGK data from [20] for Maxwell interactions are also presented in Fig. 9 for comparison. For the mass flow rate, the so-called Knudsen minimum is clearly seen around $K_{D}=1$. As some work due to the applied force is dissipated by viscous heating, different predictions of the temperature rise may affect those of mass flow rate. A higher temperature means more work has been converted to internal energy rather than kinetic energy via viscous heating. Therefore, in comparison to the ES-BGK model, the BGK model tends to underestimate the mass flow rate in the continuum regime as it overestimates the viscous effect ( see the curves for $g_{x}=1$ ). For larger Knudsen numbers, it appears that the mass flow rate is only slightly influenced by the molecular interaction detail and the Prandtl number in the simulated range, although the heat flow rates appear to be moderately influenced by them. The heat flow rate may also become negative for some Knudsen numbers, which shows the complex characteristics of forcedriven flows.

To further investigate the accuracy of the ES-BGK model, we completed more simulations for the full Boltzmann equation for $g_{x}=0.22$. As has been shown, the ES-BGK model can give satisfactory predictions of mass flow rate for Knudsen numbers up to 2. For larger Knudsen numbers, compared with the solutions of the full Boltzmann equation, the error becomes larger. The trend accords with the predictions for velocity, where the error becomes larger for larger Knudsen numbers (cf. Fig. 4). While the heat flow rates are very small in the simulated Knudsen number range, the absolute errors are not significant.

\section{Concluding remarks}

We have investigated the ES-BGK model for predicting a force-driven Poiseuille flow. It was shown that the ES-BGK model does improve predictions of the temperature field, in comparison with the BGK model, in the slip flow regime. However, the improvement is not significant for flows in the transition regime. At high Knudsen numbers, its prediction of the temperature field is even worse than that from the BGK model (in comparison with the benchmark DSMC data). Its accuracy is also worse than the S-model as shown by numerical comparisons. Alongside the previous investigation of Couette flow [8], it may now be concluded that the ES-BGK model does not always accurately capture the viscous heating effect in wall-bounded flows in the transition flow regime. It appears that this inaccuracy can also influence predictions of the mass flow rate.

As kinetic models attempt to use simple relaxation terms to capture the effect of molecular collisions, it may be no surprise to observe that they actually fail to predict the viscous heating effect at larger Knudsen numbers where the detail of molecular collisions becomes important. However, if the viscous heating is negligible the ES-BGK model may be able to perform well. In fact, the ES-BGK model still predicts reasonable velocity profiles even if the viscous heating effect is significant (e.g., the case of $g_{x}=1$ ). With its sound theoretical foundation (the H theorem), the ES-BGK model could be useful for flows in which thermal conduction plays a major role, and may also give more confidence on numerical stability.

We have provided profiles of macroscopic quantities and mass/heat flow rates from the ES-BGK model for force-driven Poiseuille flows over a range of Knudsen numbers (density, velocity, temperature and shear stress for Knudsen numbers $\left(K_{D}\right)$ between 0.1 and 1.5 , mass and heat flow rates for $\left.0.05<K_{D} \leqslant 10\right)$. The model can capture the bimodal temperature distribution phenomenon that occurs at intermediate Knudsen numbers. The Knudsen minimum is also clearly identified. As some work due to the applied force is dissipated by viscous heating, the mass flow rate does not increase linearly as the body force increases. The heat flow parallel to the wall surfaces varies in a complicated way as the Knudsen number and body force increase. In some cases it may flow in a direction opposite to the body force, the BGK model and the BE also show similar behavior. When the Knudsen number and the body force magnitude become sufficiently large, the heat flow rate tends to increase quickly with the Knudsen number.

\section{Acknowledgments}

The authors thank Nicolas Hadjiconstantinou of MIT, USA, who performed the DSMC simulations and kindly provided us the data for $K_{D}=0.05,0.1$ and 1 . This work was financially supported by the UK's Engineering and Physical Sciences Research Council under Grants No. EP/I036117/1, and by the European Community's Seventh Framework Programme FP7/ 2007-2013 under Grant agreement ITN GASMEMS No. 215504.

\section{References}

[1] G. Karniadakis, A. Beskok, N. Aluru, Microflows and nanoflows: fundamentals and simulation, Interdisciplinary Applied Mathematics, first ed., Springer, 2005.

[2] H. Struchtrup, Macroscopic transport equations for rarefied gas flows: approximation methods in kinetic theory, Interaction of Mechanics and Mathematics, Springer, 2005.

[3] P.L. Bhatnagar, E.P. Gross, M. Krook, A model for collision processes in gases. I: Small amplitude processes in charged and neutral one-component systems, Phys. Rev. 94 (3) (1954) 511-525.

[4] L.H. Holway, New statistical models for kinetic theory: methods of construction, Phys. Fluids 9 (9) (1966) 1658-1673.

[5] P. Andries, P.L. Tallec, J.-P. Perlat, B. Perthame, The Gaussian-BGK model of Boltzmann equation with small Prandtl number, Eur. J. Mech. B - Fluids 19 (6) (2000) 813-830.

[6] P. Andries, B. Perthame, The ES-BGK model equation with correct Prandtl number, in: T.J. Bartel, M.A. Gallis (Eds.), Rarefied Gas Dynamics: 22nd International Symposium, 585 (1), Springer, 2001, pp. 30-36.

[7] I. Graur, A. Polikarpov, Comparison of different kinetic models for the heat transfer problem, Heat Mass Transfer 46 (2) (2009) $237-244$.

[8] L. Mieussens, H. Struchtrup, Numerical comparison of Bhatnagar-Gross-Krook models with proper Prandtl number, Phys. Fluids 16 (8) (2004) 27972813. 
[9] M.A. Gallis, J.R. Torczynski, Investigation of the ellipsoidal-statistical Bhatnagar-Gross-Krook kinetic model applied to gas-phase transport of heat and tangential momentum between parallel walls, Phys. Fluids 23 (3) (2011) 030601.

[10] P. Andries, J.-F. Bourgat, P. le Tallec, B. Perthame, Numerical comparison between the Boltzmann and ES-BGK models for rarefied gases, Comput. Methods Appl. Mech. Eng. 191 (31) (2002) 3369-3390.

[11] D.P. Giddens, A.B. Huang, V.Y.C. Young, Evaluation of two statistical models using the shock structure problem, Phys. Fluids 14 (12) (1971) 2645.

[12] B.M. Segal, Shock-Wave Structure using nonlinear model Boltzmann equations, Phys. Fluids 15 (7) (1972) 1233.

[13] V.I. Zhuk, V.A. Rykov, E.M. Shakhov, Kinetic models and the shock structure problem, Fluid Dyn. 8 (4) (1973) $620-625$.

[14] J. Montanero, V. Garzó, Nonlinear Couette flow in a dilute gas: comparison between theory and molecular-dynamics simulation, Phys. Rev. E 58 (2) (1998) 1836-1842.

[15] V. Garzó, A. Santos, Comparison between the Boltzmann and BGK equations for uniform shear flow, Physica A 213 (1995) $426-434$.

[16] V. Garzó, M. López de Haro, Nonlinear transport for a dilute gas in steady Couette flow, Phys. Fluids 9 (3) (1997) 776.

[17] G.A. Bird, Monte Carlo simulation of gas flows, Annu. Rev. Fluid Mech. 10 (1) (1978) 11-31.

[18] E.M. Shakhov, Approximate kinetic equations in rarefied gas theory, Fluid Dyn. 3 (1) (1968) 112-115.

[19] E.M. Shakhov, Generalization of the Krook kinetic relaxation equation, Fluid Dyn. 3 (5) (1968) 95-96.

[20] K. Aoki, S. Takata, T. Nakanishi, Poiseuille-type flow of a rarefied gas between two parallel plates driven by a uniform external force, Phys. Rev. E 65 (2) (2002) 26315.

[21] F. Sharipov, V. Seleznev, Data on internal rarefied gas flows, J. Phys. Chem. Ref. Data 27 (1998) 657-706.

[22] W.H. Press, S.A. Teukolsky, W.T. Vetterling, B.P. Flannery, Numerical Recipes: The Art of Scientific Computing, third ed., Cambridge University Press, 2007.

[23] M.M. Mansour, F. Baras, A.L. Garcia, On the validity of hydrodynamics in plane Poiseuille flows, Physica A 240 (1997) $255-267$.

[24] P. Taheri, M. Torrilhon, H. Struchtrup, Couette and Poiseuille microflows: analytical solutions for regularized 13-moment equations, Phys. Fluids 21 (2009) 017102.

[25] M. Tij, A. Santos, Perturbation analysis of a stationary nonequilibrium flow generated by an external force, J. Stat. Phys. 76 (1994) $1399-1414$.

[26] M. Tij, M. Sabbane, A. Santos, Nonlinear Poiseuille flow in a gas, Phys. Fluids 10 (1998) 1021.

[27] C. Mouhot, L. Pareschi, Fast algorithms for computing the Boltzmann collision operator, Math. Comput. 75 (2006) 1833-1852.

[28] L. Wu, C. White, T.J. Scanlon, J.M. Reese, Y. Zhang, Deterministic numerical solutions of the Boltzmann equation using the fast spectral method, J. Comput. Phys. 250 (2013) 27-52. 